\title{
Experimental Characterisation of Parameters Controlling the Compressive Failure of Pultruded Unidirectional Carbon Fibre Composites
}

\author{
O.T. Thomsen ${ }^{1, ~ a, ~ K . K . ~ K r a t m a n n ~}{ }^{2, b}$ \\ ${ }^{1}$ Department of Mechanical and Manufacturing Engineering, Aalborg University, \\ Pontoppidanstræde 101, DK-9220 Aalborg East, Denmark \\ ${ }^{2}$ Fiberline Composites A/S, Barmstedt Alle 5, DK-5000, Middelfart, Denmark \\ aott@me.aau.dk, ${ }^{\mathrm{b}}$ kkr@fiberline.com
}

Keywords: UD CFRP, Pultrusion, Compressive failure, Kinkband formation models, Fourier transform misalignment analysis (FTMA), Digital Image Correlation (DIV), modified losipescu test

\begin{abstract}
The classical kink-band formation models predict that the compressive strength of UD carbon fibre reinforced composite materials (UD CFRP) is governed by fibre misalignment as well as of the mechanical shear properties. A new image analysis procedure for experimental determination of the fibre misalignment, the Fourier transform misalignment analysis (FTMA), has been developed. Moreover, a modified asymmetric Iosipescu test specimen geometry has been developed and validated for accurate measurement of the composite material shear properties without parasitic effects due to axial splitting. In the test procedure the shear strain distribution is measured using Digital Image Correlation (DIC) and the results calibrated based on FEA modelling results. Using the measured properties as input, the predictions of the classic compressive strength models have been compared with measured compressive strengths. Finally, an alternative approach to the classical kink band equilibrium has been proposed and demonstrated to provide more accurate predictions than the classical models.
\end{abstract}

\section{Introduction}

Generally the failure of composites is a complex mix of competing failure mechanisms. This is because of the in-homogeneity of the composite materials where failure can occur in any of the constituents, their interfaces and by interaction between them. Depending on constituents, interfaces and loading scenarios, different failure mechanisms may be lead to failure of the material. An overview of the most common compressive failure mechanisms in unidirectional (UD) fibre composite materials is presented in Fig. 11, Hahn and Williams [1] and Fleck [2].

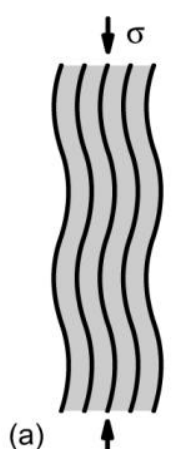

(a)

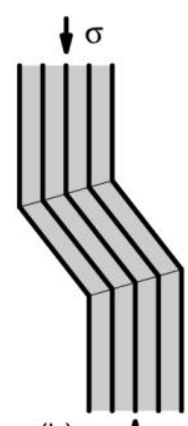

(b) $\mathbf{\uparrow}$

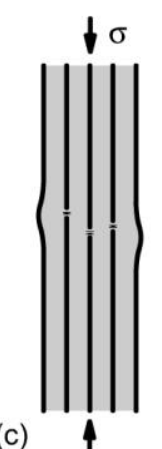

(c) $\uparrow$

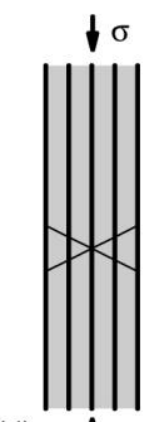

(d) $\mathrm{t}$

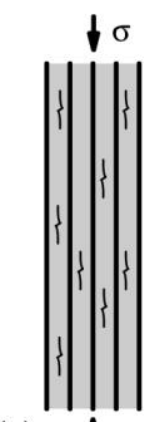

(e) $\mathbf{t}$

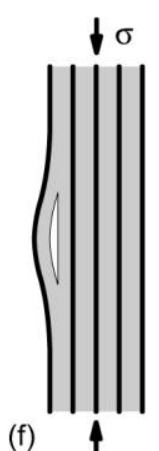

(f) 4

Fig. 1. Compressive failure modes of fibre composites. (a) Elastic micro buckling. (b) Fibre kinking. (c) Fibre crushing. (d) Shear band formation. (e) Matrix cracking. (f) Buckle delamination.

The competition between the failure mechanisms can be visualised by failure mode maps as the one shown in Fig. 2, Fleck [2]. 


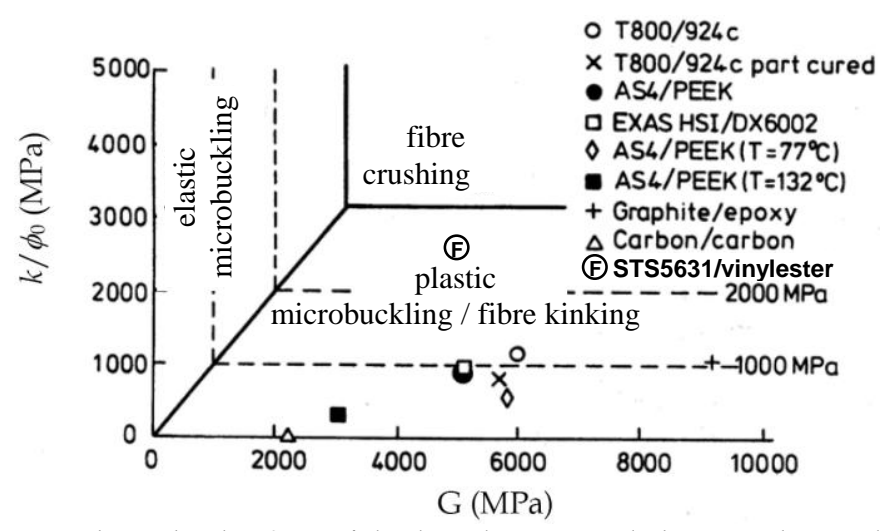

Fig. 2. Failure mode map by Fleck [2], with the shear modulus $G$ along the first axis and shear strength $k$ normalised with respect to the fibre misalignment $f_{o}$ on the second axis.

Fiberline's pultruded CFRP lamella have been added marked with $\circledast$.

As can bee seen from Fig. 2, the primary parameters governing the compressive strength of UD composites are the fibre misalignment angle $f_{o}$, the shear strength $k$, and the shear modulus $G$ of the composite material. It is seen that a very small values of $f_{o}$ compared $k$, and a $G$ value below $3 \mathrm{GPa}$ will trigger elastic microbuckling. By increasing the fibre misalignment the failure mode will switch to plastic micro buckling / fibre kinking. The shear modulus $G$ can then be increased by changing or modifying the resin system, which might also improve the shear strength. If so the failure mode can switch to fibre crushing. Most engineering composites fail by plastic microbuckling / fibre kinking. This is also the case for the vinylester based UD CFRP lamella manufactured by Fiberline Composites A/S. This has been verified by simple 3-point bending tests, and a micrograph from the failure zone is shown in Fig. , from which fibre kink band formation is clearly seen.

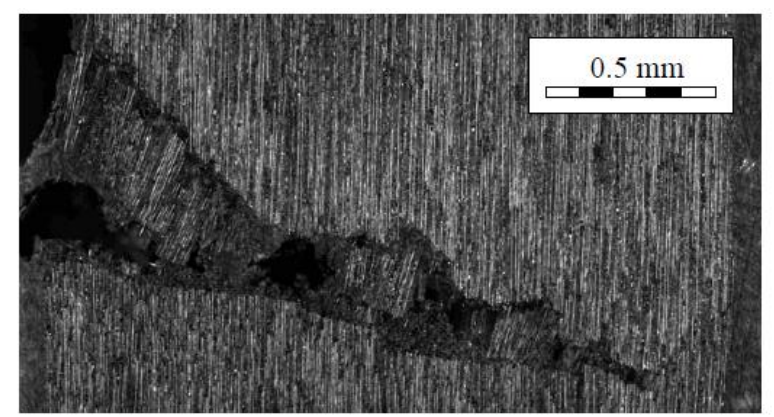

Fig. 3. Post mortem micrograph of UD CFRP tested in 3 point bending, showing kink band formations on the compressed side (left).

This work has focused on the on the elastic perfectly plastic kink band model by Budiansky [3], where the critical compressive stress $\mathbf{s}_{\mathrm{c}}$ can be determined as:

$$
\sigma_{c}=\frac{\tau_{y}}{\gamma_{y}+\phi_{0}}
$$

where $\tau_{y}$ is the shear yield stress, $\gamma_{y}$ is the shear yield strain, and $f_{o}$ is the initial fibre misalignment. Assuming linear elastic behaviour in shear (i.e. $G=\tau_{y} / \gamma_{y}$ ) Eqn. (1) can be formulated in terms of the shear modulus $G$.

$$
\sigma_{c}=\frac{G}{1+\phi_{0} / \gamma_{y}}
$$

Eqns. (1) and (2) are often written in the same equation and both referred to as the elastic perfectly plastic model. 
The elastic perfectly plastic kink band model has also been the subject of several suggestions for improvements, including Budiansky and Fleck [4] who extended the model to incorporate strain hardening, kink band inclination, combined external compressive and shear loading, and finally finite fibre stiffness. However, but the simple perfectly plastic formulation [3] remains a central part of recent studies by Soutis et al. [5], and Lee and Soutis [6]. [7], showing excellent agreement between measured and predicted strengths. See also Kratmann [8] for an overview. Taking the classical kink band model by Budiansky [3] as the basis for this work, the principal parameters controlling the kink band formation in UD CFRP laminates, i.e. the initial fibre misalignment $f_{o}$ and the composite material shear properties, defined by the shear modulus $G$ and the shear yield strain $\gamma_{\mathrm{y}}$. were determined experimentally. For evaluation of the models, the compressive strength was also measured.

\section{The FTMA Method - A New Method for Fibre Misalignment Measurement}

Of the parameters controlling fibre kinking, the fibre misalignment is probably the most difficult to quantify, as no standardised measuring techniques exist. Fibre waviness/misalignment is considered a manufacturing defect or imperfection, and its characteristics depend on the specific manufacturing process involved. Examples hereof are the differences between the repetitive waviness introduced by braiding or weaving, local waviness of a UD prepreg being layed out in a mould, and the random waviness in pultruded UD composites as sketched in Fig. .
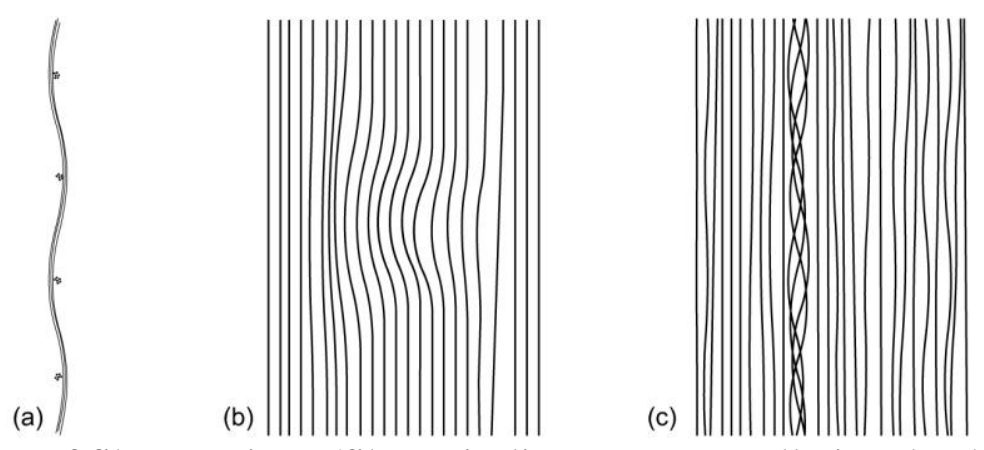

Fig. 4. Different types of fibre waviness/fibre misalignment generally involved with different types of manufacturing processes. (a) Fibre waviness from braiding and weaving. (b) Wavy regions in UD prepregs or UD tapes. (c) Random waviness in pultrusion [8].

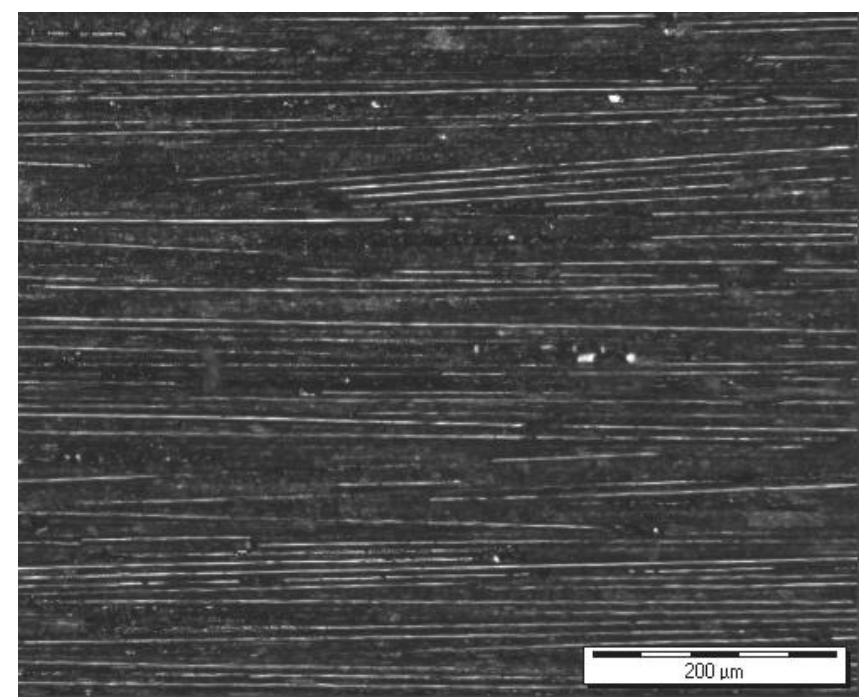

Fig. 5. Micrograph of pultruded UD CFRP manufactured by Fiberline Composites A/S. 
The pultruded UD composites studied in this work are considered to be randomly misaligned as the micrograph in Fig. 5 shows, i.e. although a few fibres follow each other; fibres next to those have a significantly different orientation. The fibre waviness/misalignment is 3-dimensional by nature. Dedicated measuring techniques developed specifically for the quantification of fibre misalignment and waviness are limited, see [9], [10], [11], [12]. In this work a novel image analysis procedure named Fourier transform misalignment analysis (FTMA) for measuring fibre misalignment in unidirectional fibre composites has been developed [13]. The FTMA method, which is a 2D technique measuring the fibre directions in section planes prepared by poslishing, measures angles of individual fibre segments isolated by Fourier noise filtering. Working with relatively low magnification micrographs (10× optical magnification) thousands of fibres can be identified, their angle measured and a statistical fibre misalignment distribution generated for the analysed plane, see Fig. 6 (sample analysis results). Areas of $30 \mathrm{~mm} \times 38 \mathrm{~mm}$ have been analysed using the FTMA method, identifying approximately 300,000 fibres in less than 30 minutes using on an Intel E8400, $2 \times 3 \mathrm{GHz}, 4 \mathrm{~GB}$ RAM running Windows XP 64-bit. From these analyses it is assessed that the FTMA method, is probably the most precise and computationally efficient analysis tool for measuring fibre misalignment that are currently available.
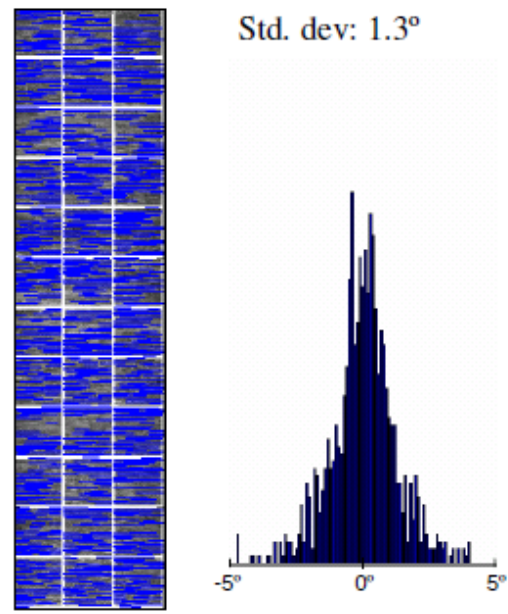

(a)

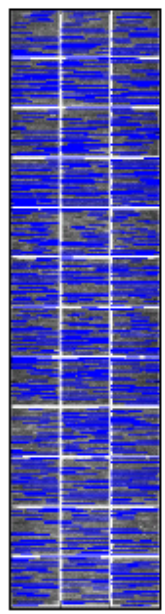

Std. dev: $0.9^{\circ}$

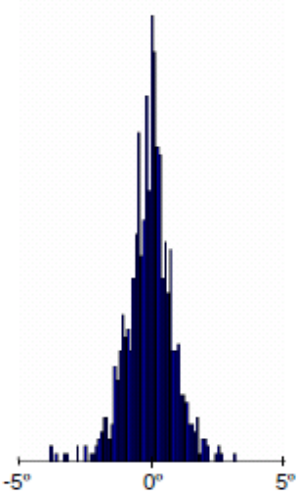

(b)

Fig. 6 FTMA of UD CFRP (a) open bath pultrusion, (b) closed injection pultrusion (Fiberline). The histograms represent the distribution of single fibre orientations, with the mean orientation equal to $0^{\circ}$. The white lines in the micrographs represent the domain borders.

\section{Determination of the Composite Material Shear Properties}

The Iosipescu shear test has been used to characterise the shear properties of pultruded unidirectional carbon fibre reinforced composites. The testing was based on the ASTM standardised Iosipescu shear test [14], which was modified with respect to the geometry, see Fig. 7. The proposed asymmetric test specimen geometry with horizontally aligned fibres in combination with GFRP tabs prevents axial splitting from occurring, and thereby provides more consistent measurements with less experimental scatter. The shear modulus and shear yield strain have been determined using the modified Iosipescu shear test combined with Digital Image Correlation (DIC), see Fig. 8 (sample DIC measurements of in-plane shear strains). The conventional and the proposed Iosipescu test specimen geometries have been tested and validated using both linear and non-linear finite element analyses. The obtained results have been compared and statistically evaluated. It has been shown that the modified test specimen geometry yields the same absolute results as the conventional Iosipescu geometry, but without change in the stress distribution during the test and with less experimental scatter. For full details see [15]. 
(a)

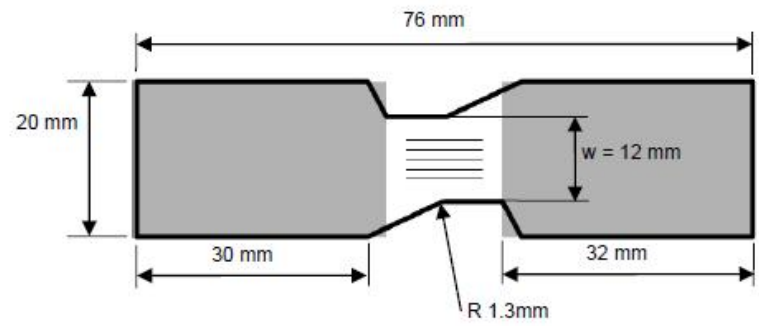

(b)

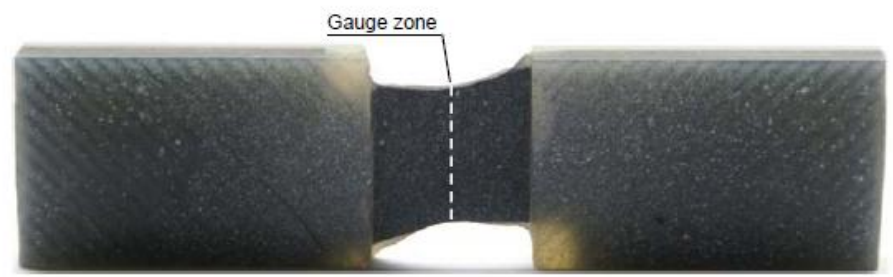

Fig. 7. Sketch with dimensions and photo of the modified asymmetric Iosipescu specimen geometry. The grey areas of the sketch indicate the tabs, and the horizontal lines in the gauge area the fibre orientation [15].

(a)
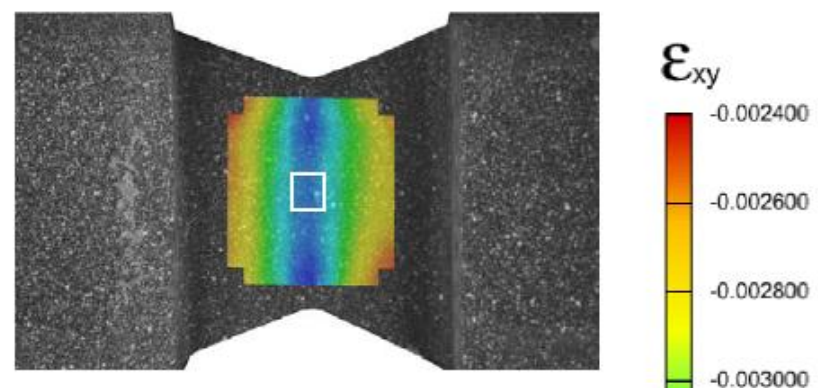

(b)
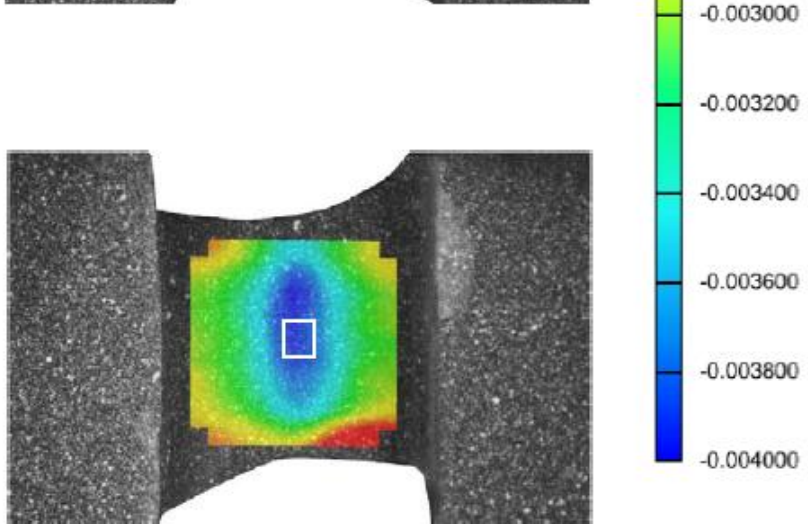

Fig. 8. (a) DIC measurement of $\varepsilon_{x y}$ distribution for tabbed symmetric specimen. (b) DIC measurement of $\varepsilon_{\mathrm{xy}}$ distribution for proposed tabbed asymmetric specimen. The white boxes represent the area from which the shear strain is determined as the average value [15].

\section{Determination of Compressive Strength}

Two variants of pultruded unidirectional carbon fibre reinforced polymer (UD CFRP) have been investigated experimentally [16]. The only difference between the variants was the resin system, which for one variant was a vinylester system (Var1), and for the other an improved resin system (Var2). For both material systems the fibre misalignment, shear stress-strain relationships and compressive strength have been determined experimentally. For the measurement of the compressive strength three different test methods were used. A simple four point bending test (ISO 14125 [17]) which is used by Fiberline for quality control. To supplement this Mechanical Combined Loading (MCL) compression tests [18] have been carried out by Risø DTU, National 
Laboratory for Sustainable Energy, Denmark. The MCL-test is a pure compression test, compliant with ISO 14126 [19], where the end-to-shear load ratio is kept fixed throughout the test by a mechanical mechanism. Finally, a four point bending test of a sandwich beam with skins made from the pultruded CFRP lamellae was developed specifically for this investigation [16], see Fig. 9.

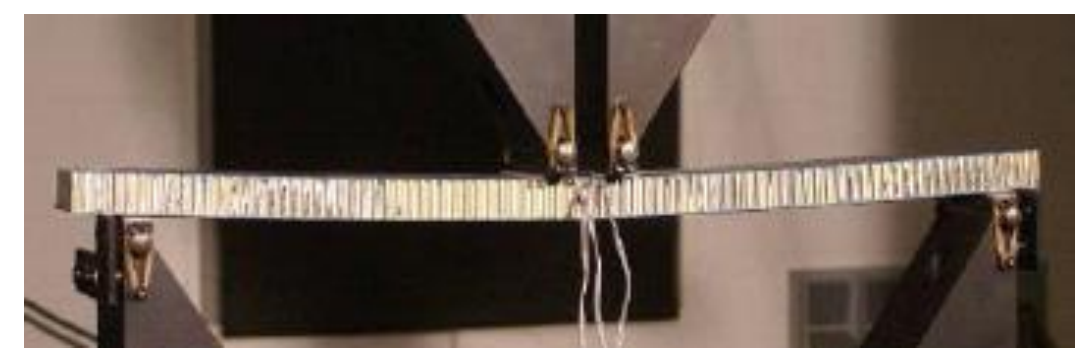

Fig. 9. Photo of the setup used for 4 point bending of UD CFRP sandwich specimens [16].

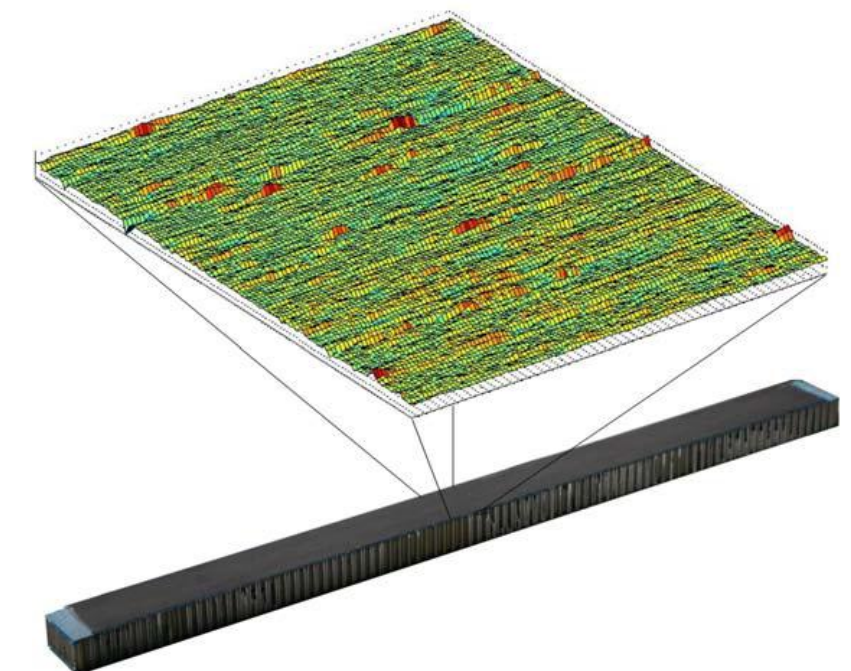

Fig. 10. Visualisation of the $2 \mathrm{D}$ variation of the domain mean angle (fibre misalignment angle) over the gauge area of a Var1 sandwich specimen.
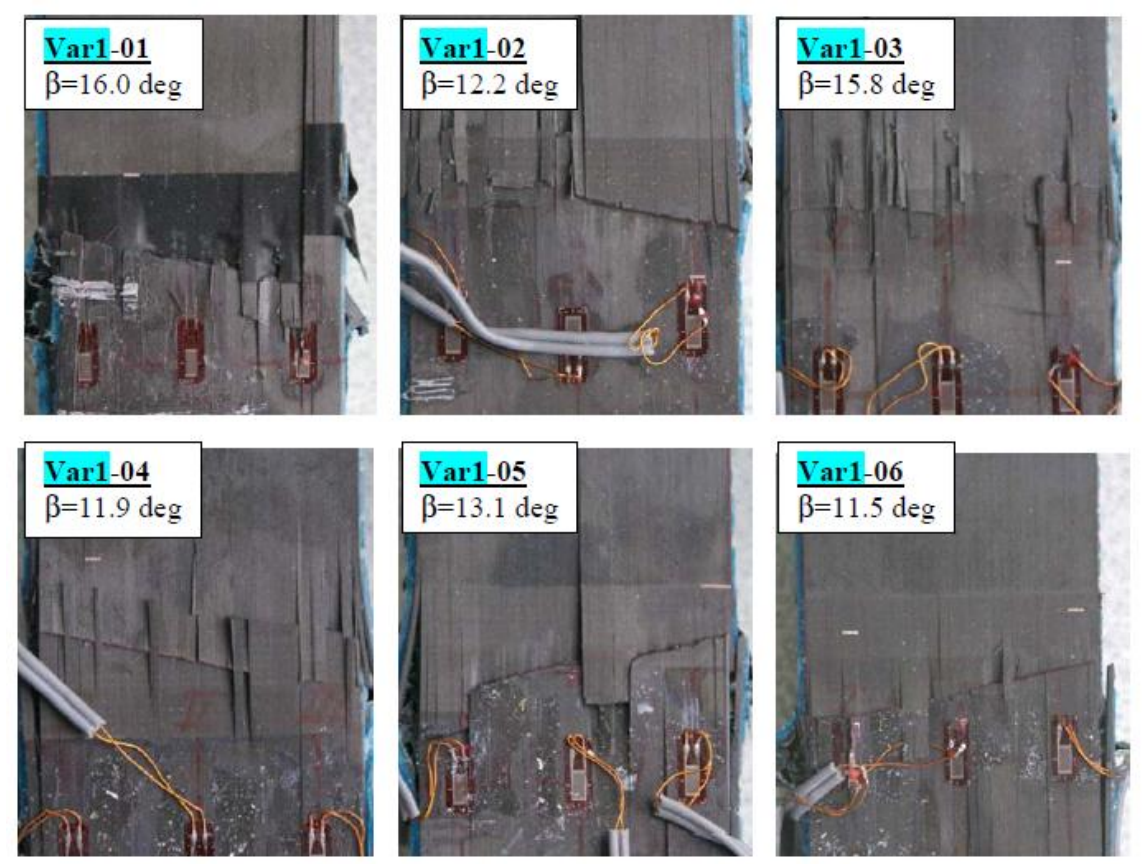

Fig. 11. Photographs of compressive kink band failure with clear inclination angle obtained by four point bending of a sandwich beam made from the Var1 material system [16]. 
It was found that the Var2 material system, which exhibits pronounced strain hardening in shear, is approximately $10 \%$ stronger than the Var1 system, which exhibits approximately elastic perfectly plastic shear behaviour. Using as input the fibre misalignment measurements based on the FTMA method (see Fig. 10 for sample visualisation of results), and the CFRP shear properties measured using the modified Iosipescu test setup, the compressive strengths were estimated. Examples of typical compressive kink band failure are shown in Fig. 11 for the Var1 material system. It was found that the classical compressive strength model by Budiansky [3] should not be used in its linearized form, and that the extended model by Budiansky and Fleck [4] also fails to predict the compressive strength, although designed to account for strain hardening. The classical compressive strength models [3], [4] are based on kink band equilibrium considerations, and an alternative approach to treat the experimental data was proposed in [16]. A good correlation with the experimentally recorded compressive strength values was found, and it was further found that transverse kink band stresses and the kink band inclination angle can not be neglected for composites exhibiting pronounced strain hardening in shear.

\section{Conclusions}

The work presented has treated the compressive failure of UD CFRP composite materials manufactured using pultrusion. Special emphasis has been focused on accurately measuring the properties governing the compressive failure, according to the classical strength models by Budiansky [3] and Budiansky and Fleck [4]. To achieve this, a new method for fast and accurate measurement of the fibre misalignment, the FTMA method, has been developed, validated and benchmarked against other existing methods. In addition, a modified Iosispescu asymmetric test specimen has been developed and validated for accurate measurement of UD composite material shear properties without parasitic effects due to axial splitting. In the test procedure the shear strain distribution is measured using Digital Image Correlation (DIC), and the results calibrated based on FEA modelling results. Finally, the predictions of the classic compressive strength models have been compared with measured compressive strengths, using the measured material properties as input. A good correlation with the experimentally obtained compressive strength values was found, and it was further found that transverse kink band stresses and the kink band inclination angle can not be neglected for composites exhibiting pronounced strain hardening in shear.

\section{Acknowledgements}

The main part of the work presented has been conducted as part of an Industrial Ph.D. programme carried out in collaboration between Fiberline Composites A/S, Denmark and the Department of Mechanical and Manufacturing Engineering, Aalborg University, Denmark, and co-sponsored by the Danish Agency for Science, Technology and Innovation.

\section{References}

[1] H.T. Hahn, J.G. Williams. Failure mechanisms in unidirectional composites. In: Composite materials: testing and design (seventh conference). ASTM STP 893. American Society for Testing and Materials, Philadelphia (1986), 115-139.

[2] N.A: Fleck NA. Compressive Failure of Fibre Composites. Advances in Applied Mechanics, Vol. 33 (1997), 43-119.

[3] B. Budiansky. Micromechanics. Computers \& Structures, Vol. 16 (1983), 3-12.

[4] B. Budiansky, N.A. Fleck. Compressive failure of fibre composites. Journal of the Mechanics and Physics of Solids; Vol. 41, (1993), 183-211

[5] C. Soutis, F.C. Smith, F.L. Matthews. Predicting the compressive engineering performance of carbon fibre-reinforced plastic. Composites: Part A, Vol. 31, (2000), 531-536. 
[6] J. Lee, C. Soutis. Thickness effect on the compressive strength of T800/942C carbon fibreepoxy laminates. Composites: Part A, Vol. 36, (2005), 213-227.

[7] J. Lee, C. Soutis. A study on the compressive strength of thick carbon fibre-epoxy laminates. Composite Science and Technology, Vol. 67, (2007); 2015-2026.

[8] K.K. Kratmann, Evaluation of Compressive Failure of Pultruded Unidirectional Carbon Fibre Composites. Ph.D. Thesis, Special Report No. 67, Department of Mechanical Engineering, Aalborg University, ISBN 87-91464-21-8 (2010).

[9] A.R. Clarke, G. Archenhold, N.C. Davidson. A novel technique for determining 3D spatial distribution of glass fibres in polymer composites. Composites Science and Technology, Vol. 55, (1995), 75-91.

[10] A.R. Clarke, G. Archenhold,, N.C. Davidson, N.A. Fleck. Determining the power spectral density of the waviness of unidirectional glass fibres in polymer composites. Applied Composite Materials, Vol. 2 (1995), 233-243.

[11] S.W. Yurgartis. Measurement of small angle fiber misalignments in continuous fiber composites. Composites Science and Technology, Vol. 30 (1987),:279-293.

[12] C.J. Creighton, M.P.F. Sutcliffe, T.W. Clyne. A multiple field image analysis procedure for characterisation of fibre alignment in composites. Composites Part A: Applied Science and Manufacturing,Vol. 32, (2001), 221-229.

[13] K.K. Kratmann, M.P.F. Sutcliffe, L.T. Lilleheden, R. Pyrz and O.T. Thomsen O.T., A novel image analysis procedure for measuring fibre misalignment in unidirectional fibre composites. Composites Science and Technology, Vol. 69, (2009), 228-238.

[14] ASTM D 5379-93. Standard Test Method for Shear Properties of Composite Materials by the V-notched Beam Method. American Society for Testing and Materials, W. Conshohocken, PA (1993).

[15] K.K. Kratmann, K.K., O.T. Thomsen, O.T., L.T. Lilleheden, R. Pyrz. Measurement of the Shear Properties of Unidirectional Carbon Fibre Composites by Using a Modified Iosipescu Test Specimen. Submitted (2010).

[16] K.K. Kratmann, K.K., O.T. Thomsen, O.T., L.T. Lilleheden, R. Pyrz. Experimental Evaluation of the Classical Compressive Strength Models for Unidirectional Carbon Fibre Composites. Submitted (2010).

[17] ISO 14125. Fibre-reinforced plastic composites - Determination of flexural properties. International Organization for Standardization, Geneva, Switzerland (1998.).

[18] J.I. Bech, S. Goutianos, T.L. Andersen, R.K. Torekov and P. Brøndsted. A new static and fatigue compression test method for composites. Strain (Early on-line), doi: 10.1111/j.14751305.2008.00521.x

[19] ISO 14126. Fibre-reinforced Plastic Composites - Determination of Compressive Properties in the In-plane Direction. International Organization for Standardization, Geneva, Switzerland. (1999) 